\title{
Effect of A-3 | 749 | delivered by glycolipid-like polymer micelles on endometriosis pain
}

\author{
This article was published in the following Dove Press journal: \\ International Journal of Nanomedicine \\ 9 November 2017 \\ Number of times this article has been viewed
}

\author{
Ming Yuan' \\ Shaojie Ding ${ }^{2}$ \\ Tingting Meng ${ }^{3}$ \\ Binbin $\mathrm{Lu}^{3}$ \\ Shihong Shao' \\ Xinmei Zhang ${ }^{2}$ \\ Hong Yuan ${ }^{3}$ \\ Fuqiang $\mathrm{Hu}^{1,3}$ \\ 'Institute of Marine Biology, Ocean \\ College, Zhejiang University, \\ Zhoushan, ${ }^{2}$ Department of \\ Gynecology, Women's Hospital, School \\ of Medicine, Zhejiang University, \\ Hangzhou, ${ }^{3}$ Institute of Pharmaceutics, \\ College of Pharmaceutical Sciences, \\ Zhejiang University, Hangzhou, \\ Zhejiang, China
}

Correspondence: Fuqiang $\mathrm{Hu}$

Department of Pharmaceutics, College

of Pharmaceutical Sciences, Zhejiang

University, 866 Yuhangtang Road,

Hangzhou 310058, China

Tel/fax+86 57l 88208439

Email hufq@zju.edu.cn

\begin{abstract}
Endometriosis is a common gynecological disease with a lack of effective clinical treatment. Current therapy often results in endometriosis pain recurrence and serious side effects. $\mathrm{P} 2 \mathrm{X}_{3}$ receptor, an adenosine triphosphate (ATP)-gated ion channel, might be implicated in endometriosis pain. In this study, chitosan oligosaccharide-g-stearic acid (CSOSA) polymer micelles-coated nanostructured lipid carriers (NLCs) were developed as a novel delivery system for A-317491, a selective $\mathrm{P}_{2} \mathrm{X}_{3}$ receptor antagonist for endometriosis pain therapy. A-317491-loaded NLC (NLC/A-317491) could be coated by CSOSA micelles to form CSOSA/ NLC/A-317491 nanoparticles. Pheochromocytoma PC12 cells, which highly expressed P2X receptors, were used as a cell model, and the CSOSA/NLC/A-317491 partly blocked the $\mathrm{Ca}^{2+}$ influx induced by ATP stimulation. In nude mouse and rat endometriotic models, CSOSA/NLC could accumulate into endometriotic lesions after vein injection. In endometriotic rats, CSOSA/ NLC/A-317491 reversed mechanical and heat hyperalgesia with long-term efficacy, which might be attributed to the massive CSOSA/NLC/A-317491 distribution in the endometriotic lesions. In conclusion, A-317491 delivered by CSOSA/NLC nanoparticles attenuated endometriosis pain in rats, and CSOSA/NLC/A-317491 could be used as an effective treatment strategy for $\mathrm{P}_{2} \mathrm{X}_{3}$-targeted therapy in endometriosis pain.
\end{abstract}

Keywords: glycolipid-like polymer, A-317491, nanostructured lipid carriers, endometriosis pain, $\mathrm{P} 2 \mathrm{X}_{3}$ receptor

\section{Introduction}

Endometriosis is a common gynecological disease defined by the presence of functional endometrium outside the uterine cavity. ${ }^{1,2}$ Pain symptoms are the main clinical manifestations of endometriosis. ${ }^{3}$ Afferent sensory fibers and proinflammatory mediators are correlated with endometriosis pain, which can be considered an inflammatory neuropathic pain. ${ }^{4-11}$ However, excision of endometriotic lesions and/or anti-inflammatory drugs cannot relieve pain symptoms completely. What is more, a high probability of recurrence and serious side effects might occur, ${ }^{1,12-15}$ thereby leading to the dilemma in its clinical treatment.

Increasing evidence indicates the importance of adenosine triphosphate (ATP) and $\mathrm{P} 2 \mathrm{X}_{3}$ receptors in endometriosis pain sensitization and transduction. Under pain pathophysiological conditions, inflammatory mediators facilitate the release of endogenous ATP to activate $\mathrm{P} 2 \mathrm{X}_{3}$, eliciting $\mathrm{Ca}^{2+}$ influx and depolarization and finally send the pain message to the central nervous system. As a selective $\mathrm{P} 2 \mathrm{X}_{3}$ receptor antagonist, A-317491 was effective in reducing neuropathic and inflammatory pain by potently blocking the $\mathrm{P} 2 \mathrm{X}_{3}$ receptor-mediated $\mathrm{Ca}^{2+}$ influx. However, the poor pharmacokinetic properties of A-317491 (short plasma half time, poor oral bioavailability, high protein 
binding, and poor distribution in endometriotic tissues) make it ineffective in endometriosis pain therapy. ${ }^{16-19}$

The drug delivery system (DDS) with significant accumulation into endometriotic tissues may serve as a feasible solution. Nanostructured lipid carriers (NLCs), consisting of solid and liquid lipids, have the advantages of improved drug entrapment efficiency (EE\%) and drug loading (DL\%) capacity for drugs with poor pharmacokinetic properties. ${ }^{20,21}$ The lipophilic characteristic of NLCs makes it easy to be captured by the reticuloendothelial system in vivo. ${ }^{22}$ Various solutions have been attempted to overcome these obstacles in recent years. In our previous study, we developed glycolipidlike polymer micelles (chitosan oligosaccharide-g-stearic acid [CSOSA]). The obtained CSOSA micelles showed excellent distribution in rat endometriotic tissues. ${ }^{23}$ Therefore, CSOSAmodified NLC may provide an option for A-317491 delivery for effective endometriosis pain therapy.

In the current study, hydrophobic A-317491 was loaded in NLC containing $20 \mathrm{wt} \%$ liquid oleic acid (NLC/A-317491). CSOSA polymers were used to modify NLC/A-317491 to form CSOSA/NLC/A-317491 nanoparticles (NPs) to improve its distribution in endometriotic lesions. The block efficacy

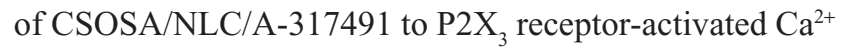
influx was investigated in pheochromocytoma cells (PC12), which had highly expressed $\mathrm{P}_{2} \mathrm{X}_{3}$. Then, the distribution of CSOSA/NLC in endometriotic tissues and the antinociceptive efficacy of CSOSA/NLC/A-317491 in endometriotic rats were measured.

\section{Materials and methods Materials, cells, and animals}

Chitosan with $\sim 19.0 \mathrm{kDa}$ average molecular weight was obtained by enzymatic degradation of $95 \%$ deacetylated chitosan (weight-average molecular weight $[\mathrm{Mw}]=450 \mathrm{kDa}$ ) and supplied by Yuhuan Marine (Yuhuan, China). Stearic acid (SA) and monostearin were provided by Shanghai Chemical Reagent Co., Ltd. (Shanghai, China). 1-Ethyl3-(3-dimethylaminopropyl) carbodiimide (EDC), ATP, and iron oxide $\left(\mathrm{Fe}_{3} \mathrm{O}_{4}, 5 \mathrm{~nm}\right)$ magnetic nanoparticles were purchased from Sigma-Aldrich Co. (St Louis, MO, USA). Octadecanoic acid (ODA) was purchased from Fluka (Sigma-Aldrich, St Louis, MO, USA). A-317491 was purchased from MedChemExpress (Shanghai, China). 1,1'-Dioctadecyl-3,3,3',3'-tetramethyl indotricarbocyanine iodide (DiR), and a fluorescent $\mathrm{Ca}^{2+}$ marker Fluo-4/AM were purchased from Thermo Fisher Scientific (Waltham, MA, USA). Trypsin, Dulbecco's Minimum Essential Medium (DMEM), penicillin, and streptomycin were purchased from Thermo Fisher Scientific. PC12 cell line was purchased from the Chinese Academy of Sciences (Shanghai, China). Nude mice and rats were purchased from Shanghai Animal Center, Chinese Academy of Sciences. All other chemicals were of analytical or chromatographic grade.

\section{Preparation of NLC and A-3 I 749 I- loaded NLC}

NLC and A-317491-loaded NLC (NLC/A-317491) were prepared by solvent diffusion. ${ }^{20}$ Monostearin $(24 \mathrm{mg}$ ) and oleic acid (OA) (6 mg) were used as lipid materials and dissolved in $3 \mathrm{~mL}$ of ethanol at $50^{\circ} \mathrm{C}$. The melted solution was dispersed into $30 \mathrm{~mL}$ of pure water containing poloxamer $188(0.1 \%, \mathrm{w} / \mathrm{v})$ under agitation at $400 \mathrm{rpm}$ in a $50^{\circ} \mathrm{C}$ water bath for $5 \mathrm{~min}$. A-317491 (3.0 mg) was mixed with the same amount of lipid materials to prepare NLC/A-317491, and the preparation method was the same as described earlier.

Fluorescein isothiocynate (FITC)-labeled ODA (ODAFITC) was synthesized as previously reported.$^{24}$ ODA-FITC $(1.5 \mathrm{mg})$ was incorporated into the same amount of lipid matrix to formulate fluorescence-labeled NLC. Additionally, $\mathrm{Fe}_{3} \mathrm{O}_{4}$ nanoparticle-loaded NLC $\left(\mathrm{NLC} / \mathrm{Fe}_{3} \mathrm{O}_{4}\right)$ was prepared to label NLC using a modified solvent diffusion method according to our previously reported method. ${ }^{25}$

\section{Preparation and characterization of CSOSA}

Chitosan oligosaccharide (CSO, $\mathrm{Mw}=19.0 \mathrm{kDa}$, degree of deacetylation: $95 \%$ ) was prepared according to our previous study. ${ }^{26}$ CSOSA was synthesized via the reaction of the carboxyl groups of SA with the amine groups of CSO in the presence of EDC. ${ }^{27}$ The proton nuclear magnetic resonance ( ${ }^{1} \mathrm{H}$ NMR) spectra of the chemicals were used to verify their chemical structures. The chemicals were dissolved in $\mathrm{CDCl}_{3}$ or $\mathrm{D}_{2} \mathrm{O}$ of $10.0 \mathrm{mg} / \mathrm{mL}$. The degrees of amino substitution of CSOSA were determined by the 2,4,6-trinitrobenzenesulfonic acid (TNBS) method, and the critical micelle concentration (CMC) was measured using pyrene as a hydrophobic fluorescent probe. ${ }^{27}$

\section{Preparation and characterization of CSOSA/NLC and CSOSA/NLC/A- 3 I 749 I nanoparticles}

Fresh NLC and NLC/A-317491 were added to the CSOSA solution with different weight ratios of CSOSA to NLC to prepare CSOSA/NLC and CSOSA/NLC/A-317491 nanoparticles. The mixture was incubated under slow horizontal shaking for $10 \mathrm{~min}$ at room temperature.

The amount of encapsulated A-317491 was detected by a high-performance liquid chromatography (HPLC) system 
(Agilent Technologies, Santa Clara, CA, USA). The binary mobile phase system consisting of reservoir A (Milli-Q water, $0.05 \%$ trifluoroacetic acid) and reservoir B (acetonitrile, $0.05 \%$ trifluoroacetic acid) was run on a gradient program (0.0 min, 70\% A and 30\% B; $8.0 \mathrm{~min}, 10 \% \mathrm{~A}$ and $90 \% \mathrm{~B}$; $13.0 \mathrm{~min}, 10 \% \mathrm{~A}$ and $90 \% \mathrm{~B} ; 13.1 \mathrm{~min}, 70 \% \mathrm{~A}$ and $30 \% \mathrm{~B}$; $16 \mathrm{~min}, 70 \% \mathrm{~A}$ and $30 \% \mathrm{~B}$ ). A flow rate of $1.0 \mathrm{~mL} / \mathrm{min}$ was used throughout the analytical run. An ultraviolet detector was set to $210 \mathrm{~nm}$. The drug EE\% and DL\% values were calculated using the following equations:

$$
\begin{gathered}
\text { EE }(\%)=\frac{\text { Weight of } \mathrm{A}-317491 \text { in NPs }}{\text { Weight of A - 317491 in feed }} \times 100 \% \\
\text { DL }(\%)=\frac{\text { Weight of A - } 317491 \text { in NPs }}{\text { Weight of A }-317491 \text { loaded NPs }} \times 100 \%
\end{gathered}
$$

The average diameter and zeta potential of nanoparticles were determined by dynamic light scattering (DLS) using a Zetasizer (Nano-ZS90; Malvern Instruments, Malvern, UK). The morphology of nanoparticles was viewed by transmission electron microscopy (TEM; JEM-1230EX; Joel, Tokyo, Japan) before the samples were stained by phosphotungstic acid $(2 \% \mathrm{w} / \mathrm{v})$ for $30 \mathrm{~s}$.

\section{In vitro drug release}

In vitro release profiles were studied by dialyzing the NLC/ A-317491 and CSOSA/NLC/A-317491 suspensions in phosphate-buffered saline (PBS) $(\mathrm{pH} 5.8)$ containing $2 \%$ Tween- 80 at $37^{\circ} \mathrm{C}$ under horizontal shaking (60 rpm) using an incubator shaker. The solubility of A-317491 in the in vitro medium was $211.35 \mu \mathrm{g} / \mathrm{mL}$. A total of $1 \mathrm{~mL}$ of the A-317491loaded suspension at a concentration of $190 \mu \mathrm{g} / \mathrm{mL}$ was sealed in a dialysis membrane (molecular weight cut off $[\mathrm{MWCO}]=7 \mathrm{kDa}$ ) and immersed in $9 \mathrm{~mL}$ of medium, which was in keeping with the requirement of sink condition. At predetermined time intervals, $1 \mathrm{~mL}$ of medium outside of the dialysis membrane was collected and supplemented with $1 \mathrm{~mL}$ of fresh medium. The samples were filtered through a $0.22 \mu \mathrm{m}$ filter and detected by an HPLC system.

\section{Cellular uptake of CSOSA/NLC nanoparticles}

PC12 cells were cultured in DMEM, which was supplemented with fetal bovine serum $(10 \%, \mathrm{v} / \mathrm{v})$, penicillin $(100 \mathrm{U} / \mathrm{mL})$, and streptomycin $(100 \mu \mathrm{g} / \mathrm{mL})$ in a humidified atmosphere of $95 \%$ air $/ 5 \% \mathrm{CO}_{2}$ at $37^{\circ} \mathrm{C}$. CSOSA micelles were labeled with rhodamine $\mathrm{B}$ isothiocyanate (RITC) via the reactive amino group of chitosan and the isothiocyanate group of RITC to investigate cellular uptake. ${ }^{28}$ Fluorescent CSOSA/NLC was prepared by RITC-labeled CSOSA and FITC-labeled NLC. The PC12 cells $\left(1 \times 10^{5}\right.$ cells/well) were seeded onto $20 \mathrm{~mm}$ glass cover slips in a 24-well plate and incubated for $24 \mathrm{~h}$. The cells were then incubated with labeled CSOSA/NLC nanoparticles for 4, 8, and $12 \mathrm{~h}$. After $100 \mu \mathrm{L}$ of Hoechst 33342 solution was added and cells further incubated for $30 \mathrm{~min}$, the cell monolayer on the coverslip was removed and washed with PBS. Nikon A1R fluorescence microscopy was used to observe the internalization capability of the CSOSA/NLC nanoparticles.

\section{In vitro cytotoxicity studies}

In vitro cytotoxicity was measured using the 3-(4,5dimethylthiazol-2-yl)-2,5 -diphenyl tetrazolium bromide (MTT) assay. The PC12 cells were exposed to different concentrations of CSOSA and CSOSA/NLC for $48 \mathrm{~h}$. MTT solution $(20.0 \mu \mathrm{L}, 5.0 \mathrm{mg} / \mathrm{mL})$ was added, and the mixture was further incubated for $4 \mathrm{~h}$. The medium was replaced with $200 \mu \mathrm{L}$ of dimethyl sulfoxide to dissolve the purple formazan crystals. The absorbance of each well at $570 \mathrm{~nm}$ was measured by a microplate reader.

\section{Intracellular calcium measurements}

PC12 cells were incubated with free A-317491 salt and CSOSA/NLC/A-317491 (10 $\mu \mathrm{M})$. At predetermined time points, the cells were loaded with Fluo-4/AM reagent $(5 \mu \mathrm{M})$ in Hank's balanced salt solution (HBSS) at $37^{\circ} \mathrm{C}$ for $30 \mathrm{~min}$. The cells were then washed three times with HBSS to remove extracellular Fluo-4/AM. Calcium imaging recordings were observed under a Nikon A1R fluorescence microscope, and an image series was recorded at $1 \mathrm{~s}$ intervals for $10 \mathrm{~min}$, with an excitation wavelength of $494 \mathrm{~nm}$. ATP solution $(100 \mu \mathrm{M})$ was added $100 \mathrm{~s}$ after the starting of the recording. NIS-Elements Imaging Software was used to calculate the fluorescence intensity (FI) kinetics of the region of interest, and the peak increase in relative fluorescent units over the baseline fluorescence $\left(\Delta F / F_{0}\right)$ was calculated (Figure 1). ${ }^{29,30}$

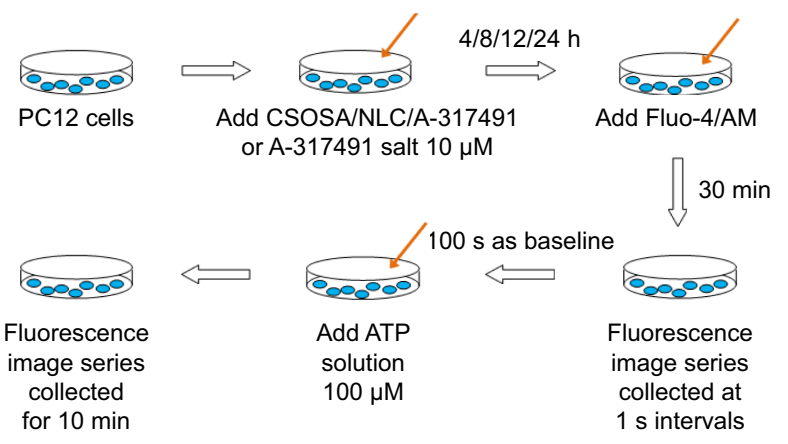

Figure I The methodology of intracellular calcium measurements. Abbreviations: CSOSA, chitosan oligosaccharide-g-stearic acid; NLC, nanostructured lipid carrier. 


\section{Surgical induction of endometriotic nude mouse and rat model}

Normal endometrium tissues were obtained from nine patients who underwent total hysterectomy with uterine fibroids. No patients received hormonal therapy in the 6 months before surgery, and all women gave their written informed consent. The study was approved by the Human Ethics Committee of the Women's Hospital, School of Medicine, Zhejiang University (ID: 20140045). Endometrial tissues were placed in Dulbecco's Modified Eagle Medium/ Nutrient Mixture F-12 (DMEM/F12) culture medium and cut into $5 \mathrm{~mm}^{2}$ fragments. Each female nude mouse (weighing 18-20 g) was implanted subcutaneously with endometrial fragments in the flank under general anesthesia and then treated with $17-\beta$ estradiol $(1 \mathrm{mg} / \mathrm{kg})$ via intramuscular injection every 5 days. The mice were tested for drug distribution 10 days after surgery. ${ }^{23,31}$

The rats were anesthetized before making a vertical incision on the abdomen. The distal $1 \mathrm{~cm}$ segment of the uterine horn was extracted and immediately placed in sterile saline. The uterine segment was cut into four pieces $\left(2 \mathrm{~mm}^{2}\right)$. Two pieces were sewn around mesenteric arteries, and the remaining two pieces were sewn on each side of the peritoneum. A total of 17 rats were induced with endometriosis, out of which two rats were sacrificed to confirm the disease 2 weeks after the surgery. After sacrifice, each cyst was excised and confirmed by hematoxylin-eosin staining and histopathology. Then, the endometriotic rats were randomly divided into three different groups, each consisting of five rats. All experimental animals were housed in a barrier facility at a monitored ambient temperature of $22^{\circ} \mathrm{C}$ in regulated 12:12 h light-dark cycles. All animals were maintained in accordance with the National Institutes of Health Guide for the Care and Use of Laboratory Animals, and the Experimental Animal Ethics Committee of Zhejiang University approved this study (ID: ZJU20160192).

\section{In vivo distribution}

Near-infrared dye DiR was encapsulated in CSOSA, CSOSA/ NLC, and NLC nanoparticles to investigate in vivo distribution. The DiR-loaded CSOSA/NLC, NLC, and CSOSA were injected into the tail vein of the endometriotic nude mouse models. The mice were observed by Maestro in vivo Imaging System (CRI Inc., Woburn, MA, USA) at predetermined time. The mice were euthanized at the end of the experiment. Various tissues, including ectopic endometrium (EcEM), were collected, weighed, and observed by the in vivo imaging system. The FI, corresponding to the amount of the nanoparticles, was read by the imaging system.
We also investigated the distribution of CSOSA/NLC in the endometriotic rat models. The FITC-labeled CSOSA/NLC nanoparticles were injected into the rats via vena caudalis. The rats were sacrificed 8 and $24 \mathrm{~h}$ later, and ectopic endometrial tissues were collected and embedded at optimal cutting temperature and frozen in liquid nitrogen vapor for further histological examination. The tissues were sectioned $(20 \mu \mathrm{m}$ thick), counterstained with 4',6-diamidino-2-phenylindole, imaged, and analyzed with VS120 Virtual Slide Microscope (Olympus, Tokyo, Japan).

\section{Animal treatment}

Control or endometriotic rats were randomly divided into three different groups, each consisting of five rats. The first group, designated as negative controls, was injected with $1.5 \mathrm{~mL}$ of sterile normal saline through their vena caudalis in the sixth week after surgical induction. The second group was given $1.5 \mathrm{~mL}$ of A-317491 salt saline solution (dose: $1.5 \mathrm{mg} / \mathrm{kg}$ ) via intravenous injection, as the positive group. The third group was treated with $1.5 \mathrm{~mL}$ of (dose: $1.5 \mathrm{mg} / \mathrm{kg}$ ) CSOSA/NLC/A-317491.

\section{Paw withdrawal test}

Mechanical and heat paw withdrawal tests were used to measure the hyperalgesia of the rats. Pain behavior was assessed before surgery, every week after surgery, and 2, 4, 8, 12, 18, and $24 \mathrm{~h}$ after drug administration. The rats were placed in a cage with a wire mesh floor and allowed to explore and groom. An electronic von Frey Anesthesiometer (Model 2390; IITC Life Science Instruments, Woodland Hills, CA, USA) with a flexible probe was applied to the plantar of right hind paws. Brisk withdrawal or paw flinching was considered positive response. The response force of the von Frey hair triggering the withdrawal of the hind paw was defined as mechanical pain threshold (MPT). The response of each rat was measured five times.

The paw withdrawal latency to noxious heat stimuli was determined with the apparatus (Model 33B; IITC Life Science Instruments). The rats were placed in a Plexiglas chamber on a glass plate. A radiant heat stimulus was applied to the heel of the right hind paw through the glass plate. The light beam was turned off when the rat lifted its foot, allowing the measurement of time between the start of the light beam and the foot lift. This time was defined as the heat source latency (HSL). Each trial was repeated five times at 5 min intervals. A cutoff time of $20 \mathrm{~s}$ was used to avoid tissue damage.

\section{Statistical analysis}

We used the Statistical Package for the Social Sciences Version 17.0 to perform statistical analyses. Nonparametric 
data were described as median (range), and parametric data were described as mean (standard error of mean). The unpaired $t$-test was used to compare the means of normal distribution among groups. $P<0.05$ was considered statistically significant $(* P<0.05 ; * * P<0.01$; $* * * P<0.001)$.

\section{Results \\ Characteristics of CSOSA/NLC nanoparticles}

The CSOSA was synthesized by a coupling reaction between the amino groups of CSO and the carboxyl group of SA. Its chemical structure was confirmed by ${ }^{1} \mathrm{H}$ NMR (Figure S1). The degree of amino substitution (SD\%) of CSOSA measured by the TNBS method was $9.3 \%$. The CMC of CSOSA was $95.5 \mu \mathrm{g} / \mathrm{mL}$ calculated according to Figure $2 \mathrm{~A}$. The average diameter of CSOSA was $67.8 \pm 7.8 \mathrm{~nm}$, with a PI of $0.27 \pm 0.01$

The CSOSA/NLC nanoparticles were prepared by mixing appropriate amounts of CSOSA and NLC and then incubating under slow horizontal shaking. The zeta potential of NLC was $-17.9 \pm 0.6 \mathrm{mV}$ (Table 1). After incubation with CSOSA, CSOSA/NLC became positively charged. CSOSA/NLC with a weight ratio of 2/1 was selected for further investigation for the requirements of cell and animal research.

The average diameters of the obtained nanoparticles are summarized in Table 1. The average diameters of CSOSA/NLC and CSOSA/NLC/A-317491 after CSOSA coating increased compared to those of NLC and NLC/A-317491. The A-317491 encapsulating efficiencies of NLC/A-317491 and CSOSA/NLC/A-317491 were $75.5 \pm 4.94$ and $64.4 \% \pm 2.57 \%$, respectively.

Figure 2B1 and $\mathrm{B} 2$ present the TEM images of CSOSA/ NLC and CSOSA/NLC/A-317491. Both the nanoparticles had spherical morphologies, and the observed sizes were close to those obtained by DLS. The structure of CSOSA/ NLC was also visualized by TEM (Figure 2B3). NLC/ $\mathrm{Fe}_{3} \mathrm{O}_{4}$ (indicated by the red arrow) was surrounded by CSOSA (indicated by the yellow arrows). This might be attributed to the positively charged CSOSA attached onto the negative surface of $\mathrm{NLC} / \mathrm{Fe}_{3} \mathrm{O}_{4}$ via electrostatic reactions.

\section{In vitro release}

The dialysis bag method was applied to study the release kinetics of A-317491 from CSOSA/NLC/A-317491 and
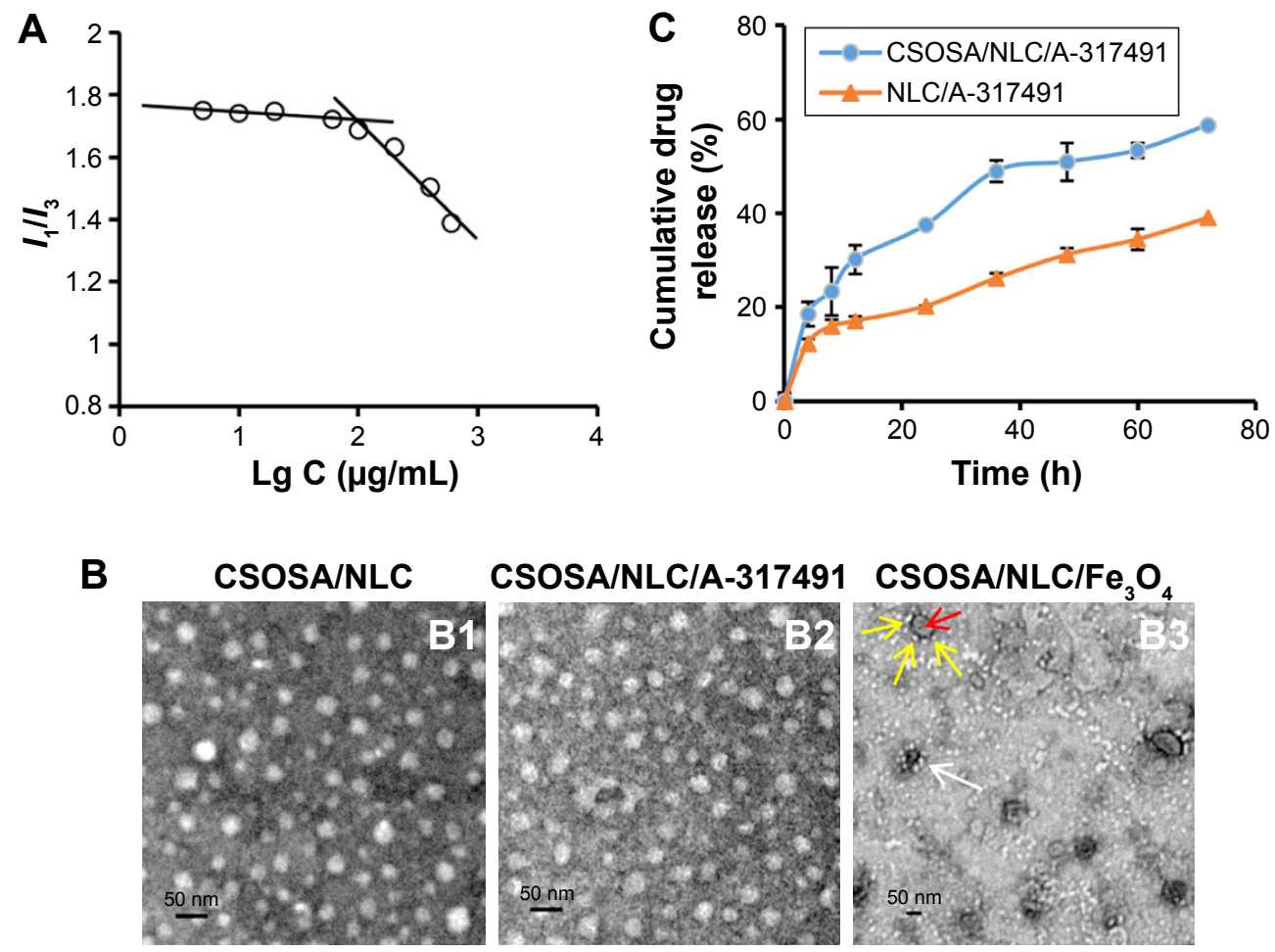

Figure 2 Characteristics of CSOSA and CSOSA/NLC nanoparticles.

Notes: (A) The $I_{1} / I_{3}$ ratio of fluorescence intensity of pyrene against logarithm concentrations (Lg C) of CSOSA. (B) TEM images of CSOSA/NLC (BI), CSOSA/NLC/A31749 I (B2), and CSOSA/NLC/Fe $\mathrm{O}_{4}$ (B3). The yellow arrows indicate the CSOSA, and the red arrow indicates the $\mathrm{NLC/Fe}{ }_{3} \mathrm{O}_{4}$. The white arrow indicates the CSOSA/NLC/ $\mathrm{Fe}_{3} \mathrm{O}_{4}$. (C) Release profiles of A-31749I from NLC/A-3I749I and CSOSA/NLC/A-31749I.

Abbreviations: CSOSA, chitosan oligosaccharide-g-stearic acid; NLC, nanostructured lipid carrier; TEM, transmission electron microscopy. 
Table I Average diameter (dn), zeta potential ( $\zeta)$, EE\%, and DL\% of the nanoparticles $(n=3)$

\begin{tabular}{llllll}
\hline Material & dn $(\mathbf{n m})$ & PI & $\zeta(\mathbf{m V})$ & EE\% & DL\% \\
\hline NLC & $27.9 \pm 1.7$ & $0.28 \pm 0.01$ & $-17.9 \pm 0.6$ & - & - \\
NLC/A-3I749I & $36.5 \pm 4.3$ & $0.27 \pm 0.01$ & $-12.8 \pm 1.0$ & $75.5 \pm 4.94$ & $7.02 \pm 0.43$ \\
CSOSA/NLC & $4 I .2 \pm 3.0$ & $0.34 \pm 0.01$ & $27.0 \pm 0.9$ & - & - \\
CSOSA/NLCI & $48.1 \pm 5.2$ & $0.22 \pm 0.02$ & $28.3 \pm 0.4$ & $64.4 \pm 2.57$ & $2.10 \pm 0.08$ \\
A-3I749I & & & & &
\end{tabular}

Note: Data presented as mean \pm standard deviation.

Abbreviations: CSOSA, chitosan oligosaccharide-g-stearic acid; DL\%, drug loading; $\mathrm{EE} \%$, entrapment efficiency; NLC, nanostructured lipid carrier; PI, polydispersity index.

NLC/A-317491 (Figure 2C). The cumulative release of A-317491 from CSOSA/NLC/A-317491 was $18.6 \% \pm 1.94 \%$ in $4 \mathrm{~h}$ and $58.8 \% \pm 1.57 \%$ in $72 \mathrm{~h}$, and that of NLC/A-317491 was $12.3 \pm 1.02$ and $39.0 \% \pm 2.22 \%$. CSOSA/NLC/A-317491 had a faster drug release rate than NLC/A-317491, which may be because of the hydrophilic modification by CSOSA polymers. The structure of CSOSA/NLC visualized by TEM is shown in Figure 2B3. NLC was surrounded by the amphiphilic glycolipid-like polymer CSOSA. We hypothesized that hydrophobic SA segments could insert into the lipophilic NLC and the hydrophilic CSO surrounded the NLC surface. Amphiphilic CSOSA functioned as a surfactant and thus facilitated the release of A-317491.

\section{Cellular uptake and in vitro cytotoxicity of CSOSA/NLC nanoparticles}

Obvious fluorescent signals were observed in PC12 cells, and fluorescent CSOSA/NLC nanoparticles showed a time-dependent increase of green fluorescence, which indicated that the CSOSA/NLC nanoparticles were effectively internalized into PC12 cells (Figure 3). The yellow fluorescence was the overlap of red fluorescence (CSOSA) and green fluorescence (NLC), indicating the co-localization of CSOSA with NLC within $12 \mathrm{~h}$.

The cytotoxicities of CSOSA/NLC and CSOSA were measured against PC12 cells. At concentration $500 \mu \mathrm{g} / \mathrm{mL}$, the percentages of survival cells for CSOSA/NLC and CSOSA were $61.3 \%$ and $53.4 \%$, respectively.

\section{Inhibitory effect of CSOSA/NLC/A- 3 I 749 I on the $\mathrm{Ca}^{2+}$ influx of $\mathrm{PCI} 2$ cells}

$\mathrm{PC} 12$ cells, highly expressing $\mathrm{P} 2 \mathrm{X}_{3}$, were used to investigate the effect of CSOSA/NLC/A-317491 on blocking P2X $\mathrm{X}_{3}-$ activated $\mathrm{Ca}^{2+}$ influx induced by ATP. The intracellular $\mathrm{Ca}^{2+}$ level was represented by the Fluo-4/AM FI. Figure 4A exhibits the fluorescence images of PC12 cells before and after ATP stimulation. PC12 cells incubated with CSOSA/ NLC/A-317491 for $12 \mathrm{~h}$ and A-317491 salt for $1 \mathrm{~h}$ showed
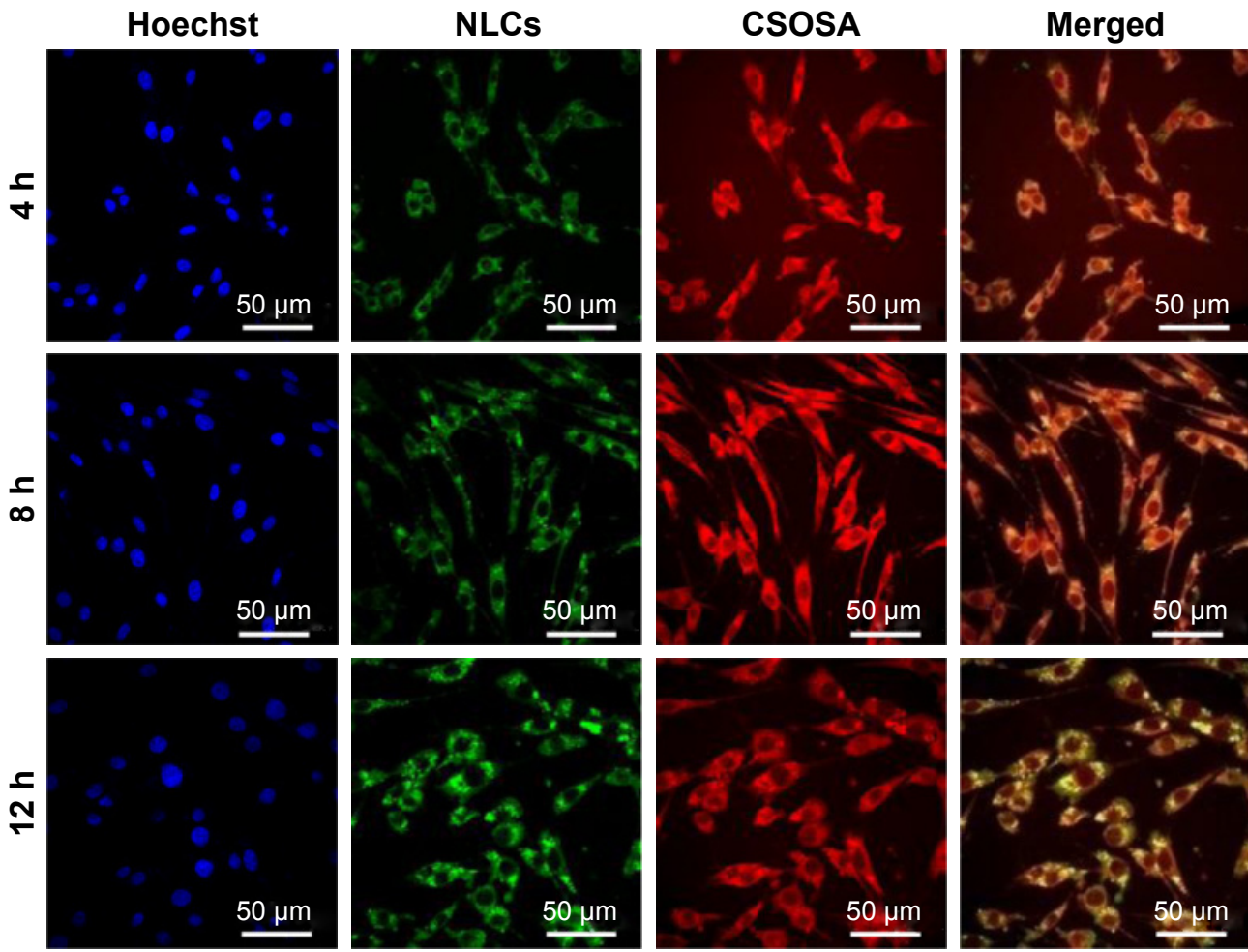

Figure 3 Cell internalization fluorescence images of CSOSA/NLC nanoparticles in PCI 2 cells within 4,8 , and 12 h.

Note: Images show the channel of the FITC-labeled NLC channel (green), the RITC-labeled CSOSA channel (red), and the Hoechst-stained cell nucleus channel (blue). Abbreviations: CSOSA, chitosan oligosaccharide-g-stearic acid; FITC, fluorescein isothiocynate; NLCs, nanostructured lipid carriers; RITC, rhodamine B isothiocyanate. 
A

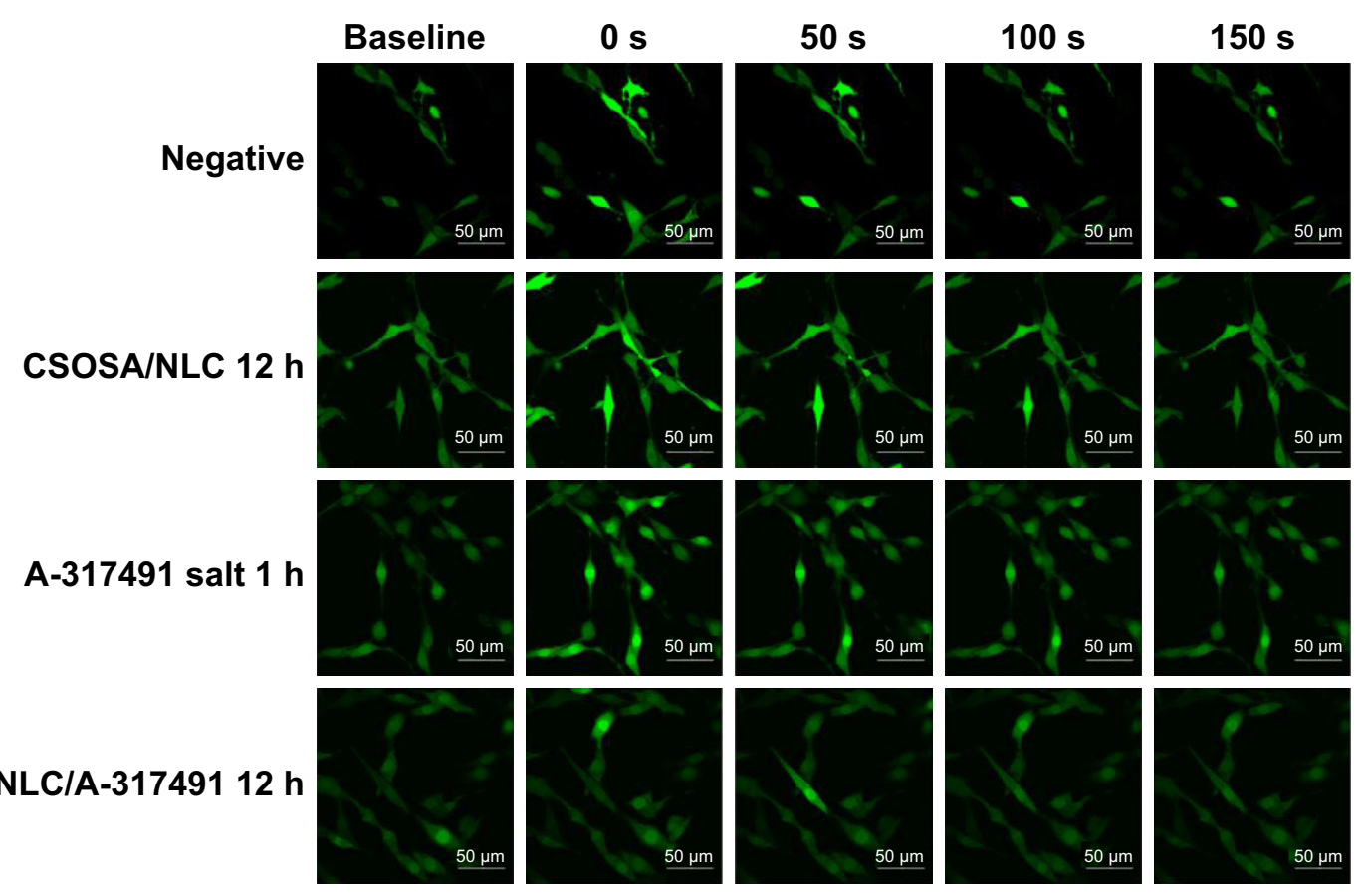

B1

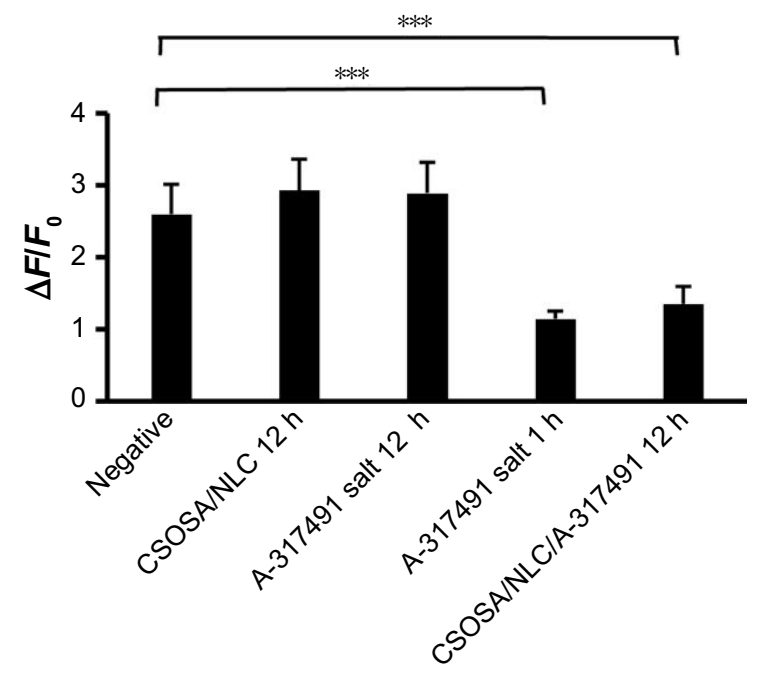

B2

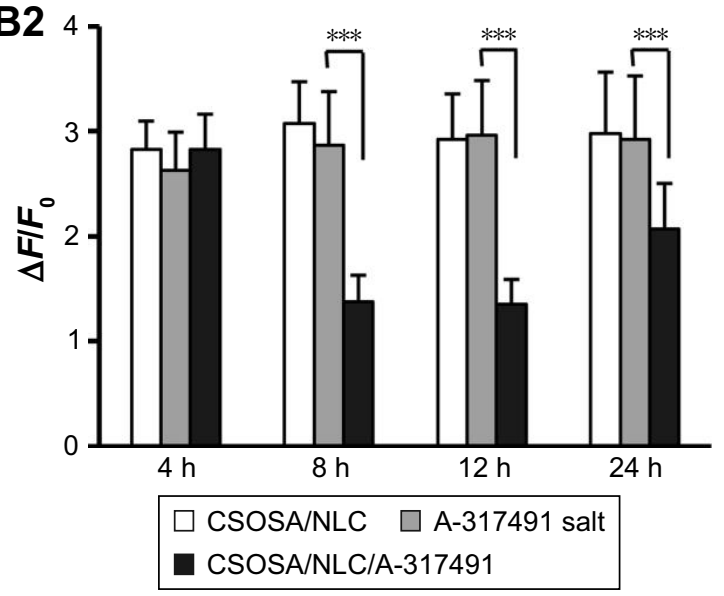

Figure 4 Inhibition of ATP-induced $\mathrm{Ca}^{2+}$ influx in $\mathrm{PCI} 2$ cells.

Notes: (A) PCI 2 cell fluorescence images of baseline (before ATP stimulation), $0 \mathrm{~s}$ (the brightest fluorescence images after ATP stimulation), $50 \mathrm{~s}$, I00 s, I50 s, and $200 \mathrm{~s}$. (BI, B2) $\mathrm{Ca}^{2+}$ peak increase $\left(\Delta F / F_{0}\right)$ in PCI2 cells incubated with A-3I749I salt, CSOSA/NLC, and CSOSA/NLC/A-3I749I for predetermined time under I00 $\mu M$ ATP stimulation. Data are expressed as the mean (SEM; $n=12$ ). $* * * P<0.001$.

Abbreviations: ATP, adenosine triphosphate; CSOSA, chitosan oligosaccharide-g-stearic acid; NLC, nanostructured lipid carrier; SEM, standard error of mean.

decreased FI compared to negative cells, which meant decreased $\mathrm{Ca}^{2+}$ influx. No significant difference was found between the negative control and CSOSA/NLC, suggesting no effect of CSOSA/NLC on $\mathrm{Ca}^{2+}$ influx.

The time course of $\mathrm{Ca}^{2+}$ fluorescence was analyzed, and the mean amplitude of calcium peaks $\left(\Delta F / F_{0}\right)$ was calculated. The lower amplitude $\left(\Delta F / F_{0}\right)$, less $\mathrm{Ca}^{2+}$ influx, meant higher efficacy of A-317491. The results are shown in Figure 4B1. The $\Delta F / F_{0}$ of the PC12 cells with
CSOSA/NLC/A-317491 incubated for $12 \mathrm{~h}(1.35 \pm 0.24)$ was comparable with that of A-317491 salt incubated for $1 \mathrm{~h}(1.14 \pm 0.11)$, and it significantly decreased compared to the negative control $(2.60 \pm 0.41)$ and CSOSA/NLC $(2.93 \pm 0.43)$.

The PC12 cells pretreated with CSOSA/NLC/A-317491 showed decreased $\mathrm{Ca}^{2+}$ influx after incubation for 8-24 h. However, after incubation for $4 \mathrm{~h}, \mathrm{~A}-317491$ salt had nearly no efficacy in blocking ATP-evoked calcium transients. 
Free A-317491 salt blocked $\mathrm{Ca}^{2+}$ influx at $1 \mathrm{~h}$ and rapidly lost its efficacy. By contrast, CSOSA/NLC/A-317491 showed prolonged, delayed efficacy after incubation from 8 to $24 \mathrm{~h}$ (Figure 4B2).

A-317491, a nonnucleotide antagonist that is highly selective over other $\mathrm{P} 2$ receptors, potently blocks $\mathrm{P} 2 \mathrm{X}_{3}$ receptor-mediated $\mathrm{Ca}^{2+}$ influx. The blocking efficacy is rapid in onset, reversible, and devoid of nonspecific effects. ${ }^{32}$ Our studies showed that free A-317491 salt attenuates the $\mathrm{Ca}^{2+}$ influx induced by ATP in PC12 cells in $1 \mathrm{~h}$ but loses its efficacy after $4 \mathrm{~h}$. While packaged by CSOSA/NLC, A-317491 still maintained its antagonism efficacy (from 8 to $24 \mathrm{~h}$ ), which showed delayed and prolonged efficacy. This might be attributed to the persistent cellular drug release after the rapid uptake of the nanoparticles.

\section{In vivo distribution}

The in vivo distributions of CSOSA/NLC, CSOSA, and NLC in EcEM were investigated with an endometriotic nude mouse model. Obvious fluorescence signals from CSOSA/DiR,
CSOSA/NLC/DiR, and NLC/DiR were observed in ectopic endometrial tissue at $8 \mathrm{~h}$ after vein injection (Figure 5A). More CSOSA/NLC accumulated into EcEM than NLC during the entire experimental process, and the distribution of CSOSA/NLC was similar to that of CSOSA. The NLC modified with CSOSA maintained the feature of CSOSA and exhibited improved distribution compared to NLC.

As shown in Figure 5B, the fluorescence signal in the EcEM was significantly brighter than that in the ovary and uterus, which indicated that the delivery systems avoided the undesired effect on the reproductive organs. The FI per gram of various tissues after $48 \mathrm{~h}$ was semiquantitatively analyzed (Figure 5C) by Maestro 2.10.0 software. The highest accumulation of CSOSA/NLC in EcEM was 1.17 times of CSOSA and 1.94 times of NLC. Compared to NLC, the modified CSOSA polymers significantly promoted the distribution of CSOSA/NLC in EcEM.

Figure 6 shows the fluorescent images of endometriotic lesions from endometriotic rats after the FITC-labeled CSOSA/NLC was injected for 8 and $24 \mathrm{~h}$. Strong green
A
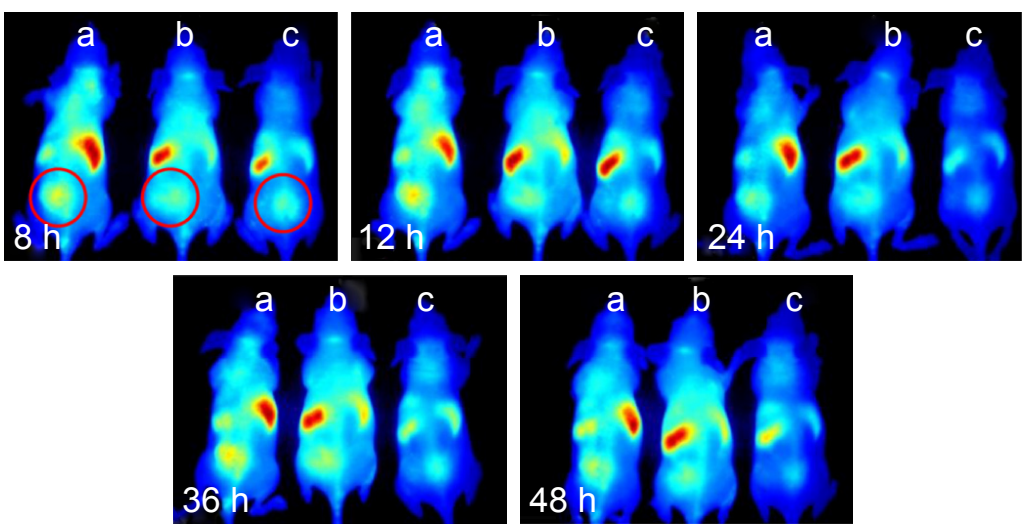

C

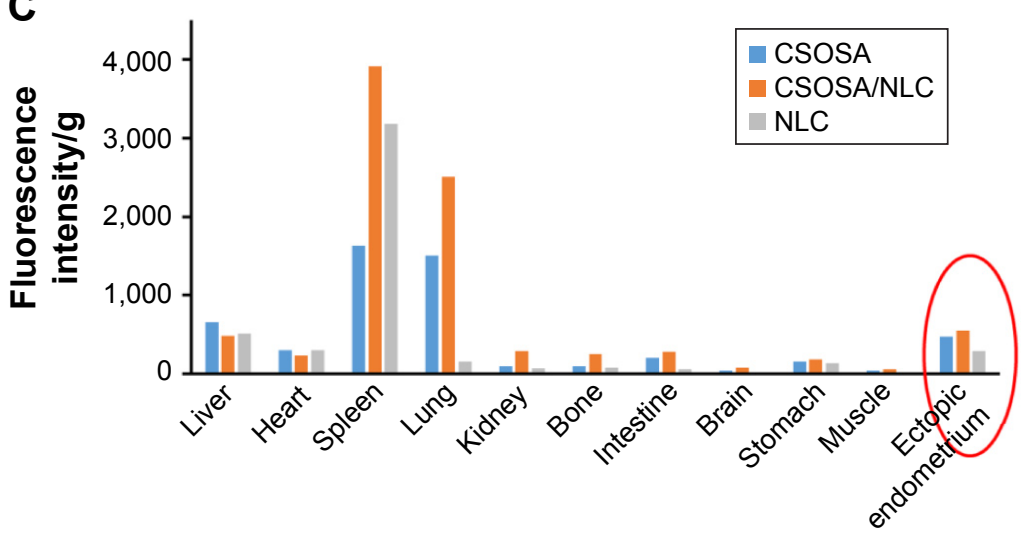

B
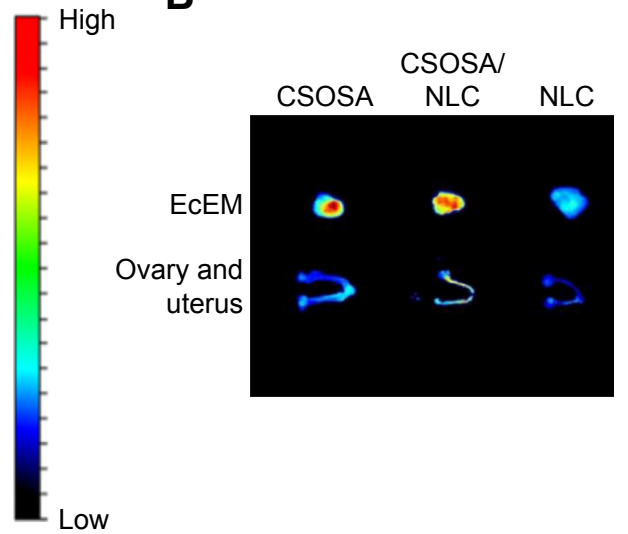

Figure 5 In vivo distribution on EcEM-bearing nude mice of CSOSA, CSOSA/NLC, and NLC.

Notes: (A) In vivo distribution on EcEM-bearing nude mice of CSOSA (a), CSOSA/NLC (b), and NLC (c) after intravenous injection for 8, 12 , 24, 36, and 48 h. (B) Fluorescent imaging of ectopic endometrium, ovary, and uterus at $48 \mathrm{~h}$ after intravenous injection of the nanoparticles in mice. (C) Accumulation of nanoparticles in various tissues calculated as FI per gram of tissue. The red circle indicates the distribution of CSOSA, CSOSA/NLC and NLC in ectopic endometrium.

Abbreviations: CSOSA, chitosan oligosaccharide-g-stearic acid; EcEM, ectopic endometrium; Fl, fluorescence intensity; NLC, nanostructured lipid carrier. 

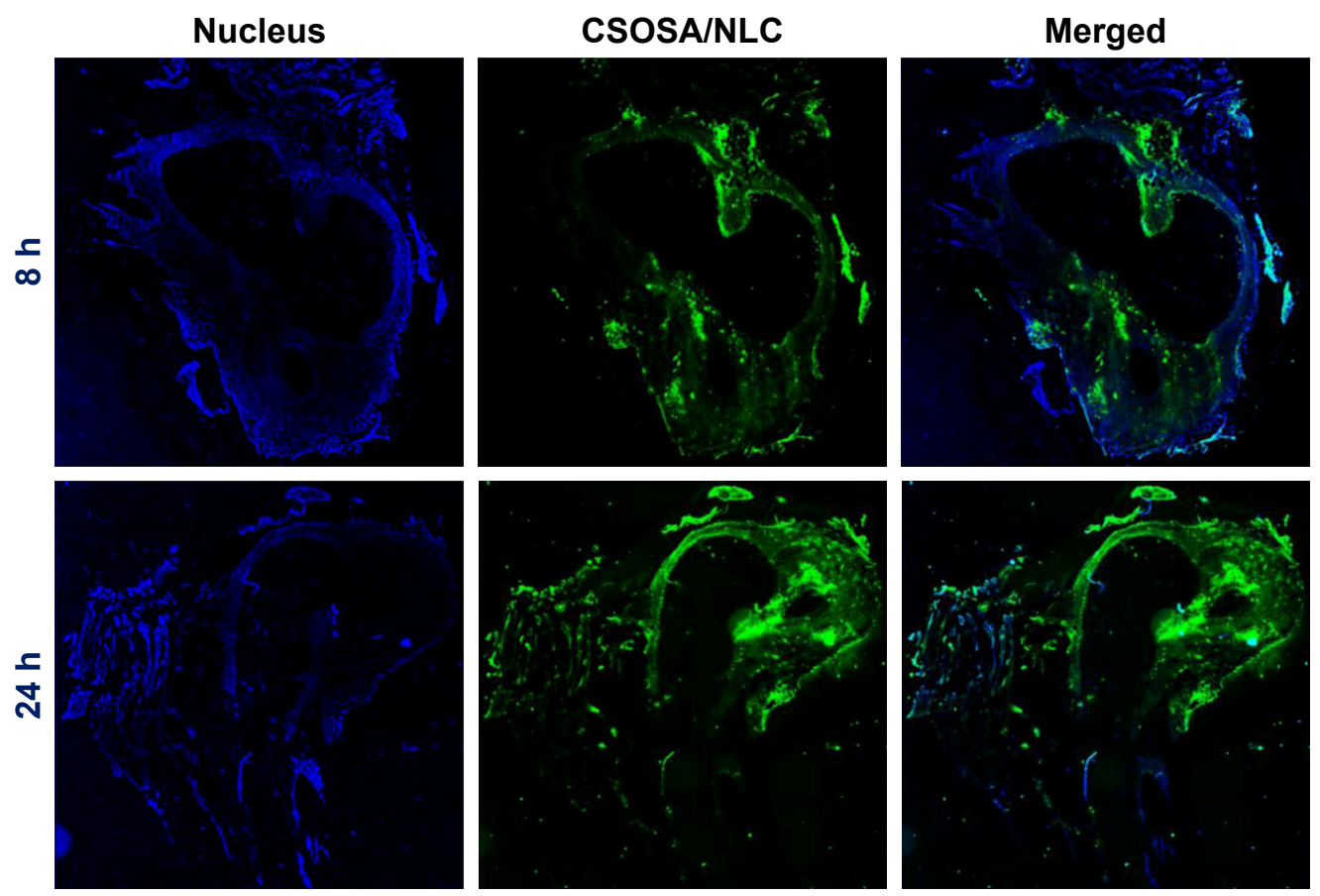

Figure 6 Fluorescent images of the frozen microtome section of EcEM observed after the model rat was intravenously injected with FITC-labeled CSOSA/NLC for 8 and 24 h.

Notes: The blue color represents nucleus, and the green color represents CSOSA/NLC. Magnification: I0X.

Abbreviations: CSOSA, chitosan oligosaccharide-g-stearic acid; EcEM, ectopic endometrium; FITC, fluorescein isothiocynate; NLC, nanostructured lipid carrier.

fluorescence was observed, which indicated that the CSOSA/ NLC could accumulate into the ectopic lesions.

\section{Inhibitory effect of CSOSA/NLC/A- 3 I749I on endometriosis pain hyperalgesia in rats}

Endometriotic surgery induced both mechanical and heat hyperalgesia in rats, and CSOSA/NLC/A-317491 treatment attenuated pain with prolonged efficacy. Low MPT or HSL represented the relative hyperalgesia. Rats with endometriotic surgery showed lower MPT/HSL compared to the control group at 21, 28, 35, and 42 days (Figure 7A and B).

CSOSA/NLC/A-317491 and A-317491 salt induced significant behavioral changes in the endometriotic group (Figure 7E and F). A-317491 salt increased MPT and HSL at 2 and $4 \mathrm{~h}$ compared to the saline group and lost its efficacy after $8 \mathrm{~h}$. By contrast, CSOSA/NLC/A-317491 showed increased MPT and HSL at 8, 12, and $18 \mathrm{~h}$, and the effect lasted to 24 h. CSOSA/NLC/A-317491 and A-317491 salt had no effect on the normal rats (Figure $7 \mathrm{C}$ and D).
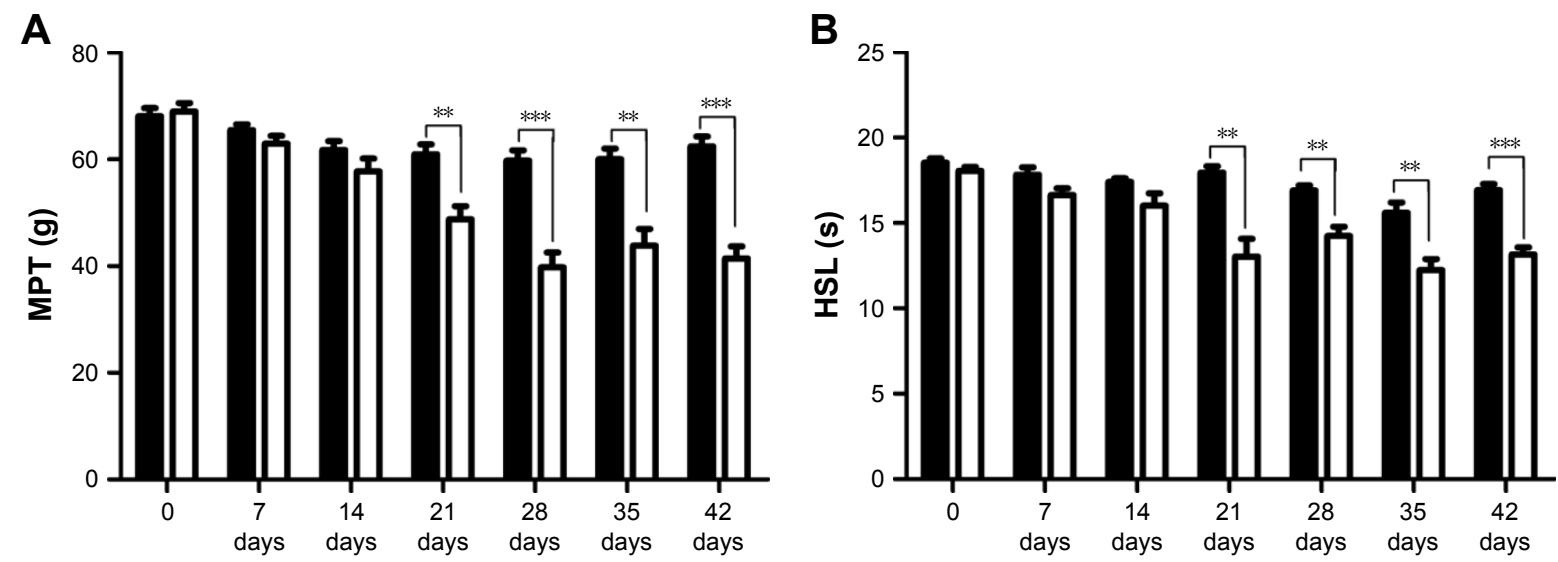

Ctrl $\square \mathrm{Em}$

Figure 7 (Continued) 

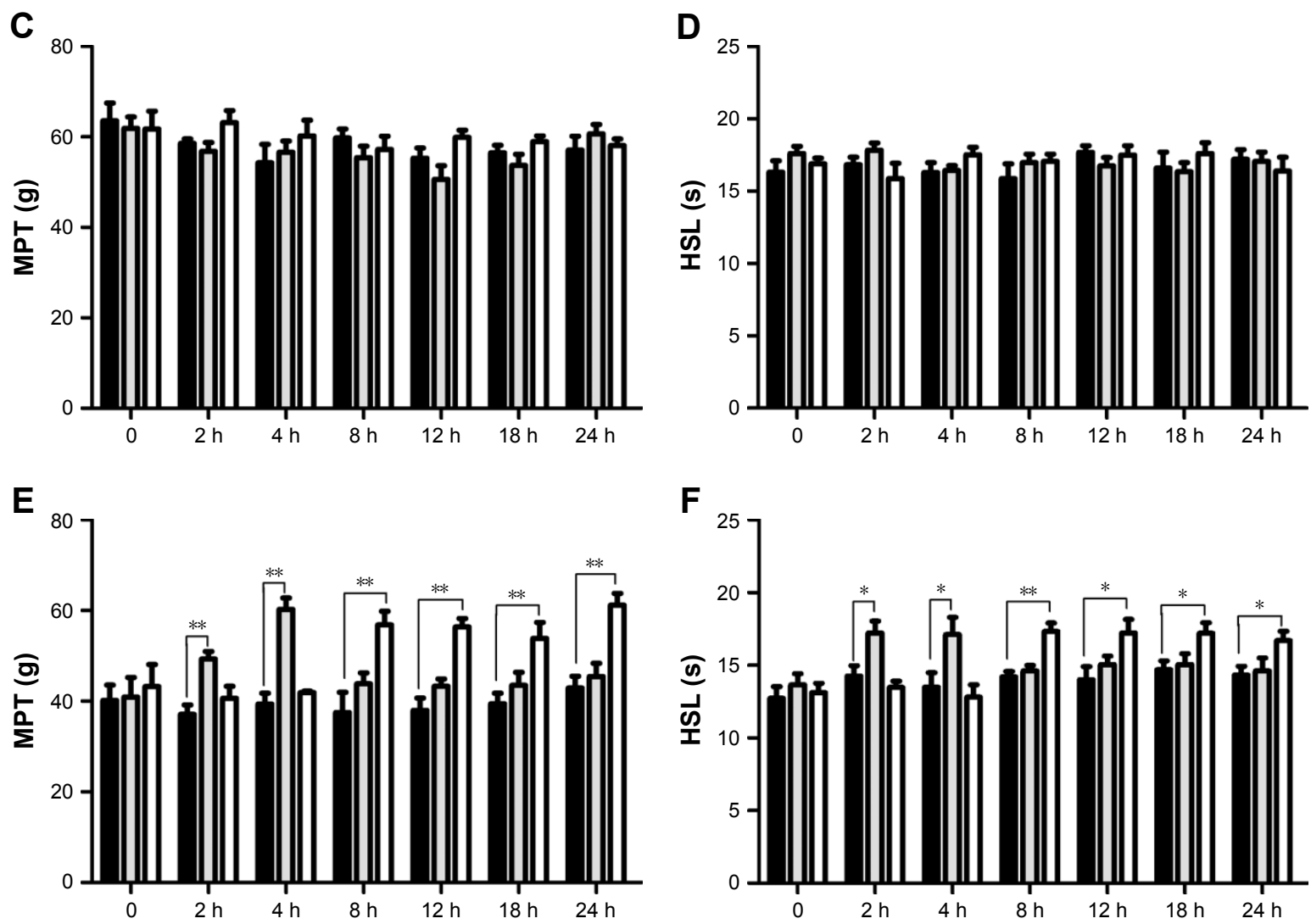

Normal saline $\square$ A-317491 salt $\square$ CSOSA/NLC/A-317491

Figure $\mathbf{7}$ Variations in pain behavior in rats.

Notes: Mechanical (A) and heat (B) hyperalgesia behaviors measured every week after endometriotic or sham surgery. (C and $\mathbf{D})$ Hyperalgesia behavior observed at 2, $4,8,12,18$, and $24 \mathrm{~h}$ after drug administration in normal rats. ( $\mathbf{E}$ and $\mathbf{F}$ ) Behavior tested after drug administration in endometriotic rats. Data are expressed as the mean (SEM; $\mathbf{A}$ and $\mathbf{B}, \mathrm{n}=15 ; \mathbf{C}-\mathbf{F}, \mathrm{n}=5$ ). $* \mathrm{P}<0.05 ; * * \mathrm{P}<0.01$; $* * * \mathrm{P}<0.00 \mathrm{I}$.

Abbreviations: CSOSA, chitosan oligosaccharide-g-stearic acid; Ctrl, control group; Em, endometriotic group; HSL, heat source latency; MPT, mechanical pain threshold; NLC, nanostructured lipid carrier; SEM, standard error of mean.

The key to endometriosis pain therapy is to ensure that the medicine can reach the endometriotic site and exhibit its antagonistic efficacy. In this study, we found that a dose of A-317491 $(1.5 \mathrm{mg} / \mathrm{kg})$ delivered by CSOSA/NLC significantly relieved the mechanical and thermal hyperalgesia of endometriotic rats and the antinociceptive effect lasted from 8 to $24 \mathrm{~h}$. These results were consistent with the in vivo distribution. The antinociceptive effect of A-317491 salt lasted from 2 to $4 \mathrm{~h}$. Consequently, the combination therapy of A-317491 salt and CSOSA/NLC/A-317491 could be considered. Different doses and longer behavior tests might be set in further study. The presented evidence may provide a reference on the manner of delivery of clinical drugs used to treat chronic pain.

Figure 8 summarizes the structure of CSOSA/NLC/ A-317491 and its inhibition process of ATP-induced $\mathrm{Ca}^{2+}$ influx. The amphiphilic glycolipid-like polymer CSOSA could self-assemble to form a positively charged core-shell structure. Then, the negatively charged NLC surface was coated with the positive chitosan chains via electrostatic interactions. This special spatial structure induced excellent internalization into cells and increased the cellular uptake of the loaded A-317491. ${ }^{33}$ A-317491, persistently released

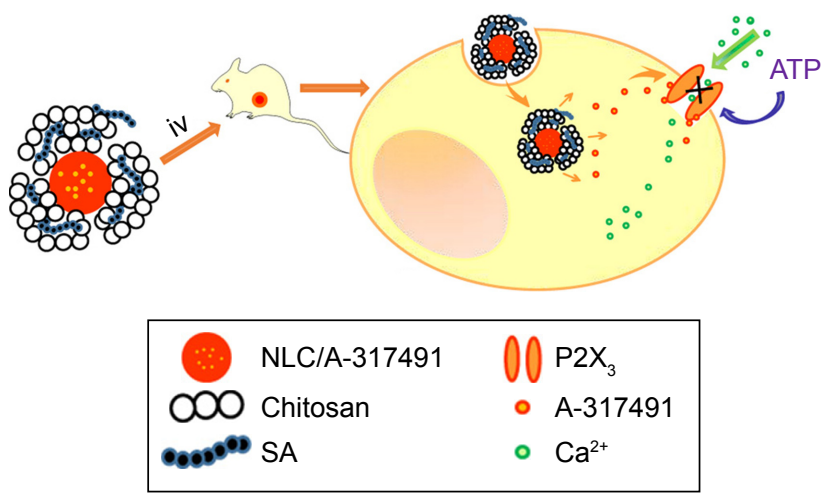

Figure 8 Schematic structure of CSOSA/NLC/A-31749I and its inhibition of ATPinduced $\mathrm{Ca}^{2+}$ influx.

Abbreviations: ATP, adenosine triphosphate; CSOSA, chitosan oligosaccharide-gstearic acid; NLC, nanostructured lipid carrier; iv, intravenously; SA, stearic acid. 
from the carriers, worked on $\mathrm{P} 2 \mathrm{X}_{3}$ receptors to inhibit $\mathrm{Ca}^{2+}$ influx induced by ATP.

\section{Conclusion}

In this research, glycolipid-like polymer coated NLCs (CSOSA/NLC) were developed, and presented excellent accumulation in the ectopic lesions of endometriotic nude mice and rats. CSOSA/NLC/A-317491 could inhibit the $\mathrm{Ca}^{2+}$ influx induced by extracellular ATP in PC12 cells and relieved the mechanical and thermal hyperalgesia of endometriotic rats for a delayed and prolonged effective time compared to A-317491 salt. Thus, CSOSA/NLC is a promising DDS for endometriosis pain therapy, suggesting a novel pathway for $\mathrm{P}_{2} \mathrm{X}_{3}$-targeted therapy in inflammatory and neuropathic pain.

\section{Acknowledgment}

We appreciate the financial support from the National Nature Science Foundation of China (nos 81473144, 81471433, and 81671429).

\section{Disclosure}

The authors report no competing financial interest in this work.

\section{References}

1. Fauconnier A, Chapron C. Endometriosis and pelvic pain: epidemiological evidence of the relationship and implications. Hum Reprod Update. 2005;11(6):595-606.

2. Moen MH, Stokstad T. A long-term follow-up study of women with asymptomatic endometriosis diagnosed incidentally at sterilization. Fertil Steril. 2002;78(4):773-776.

3. Nnoaham KE, Hummelshoj L, Webster P, et al. Impact of endometriosis on quality of life and work productivity: a multicenter study across ten countries. Fertil Steril. 2011;96(2):366-431.

4. McKinnon BD, Bertschi D, Bersinger NA, Mueller MD. Inflammation and nerve fiber interaction in endometriotic pain. Trends Endocrinol Metab. 2015;26(1):1-10.

5. Zhang X, Lu B, Huang X, Xu H, Zhou C, Lin J. Endometrial nerve fibers in women with endometriosis, adenomyosis, and uterine fibroids. Fertil Steril. 2009;92(5):1799-1801.

6. Zhang X, Yao H, Huang X, Lu B, Xu H, Zhou C. Nerve fibres in ovarian endometriotic lesions in women with ovarian endometriosis. Hum Reprod. 2010;25(2):392-397.

7. McAllister SL, Dmitrieva N, Berkley KJ. Sprouted innervation into uterine transplants contributes to the development of hyperalgesia in a rat model of endometriosis. PLoS One. 2012;7(2):e317582.

8. Sikora J, Mielczarek-Palacz A, Kondera-Anasz Z. Imbalance in cytokines from interleukin-1 family - role in pathogenesis of endometriosis. Am J Reprod Immunol. 2012;68(2):138-145.

9. Wickiewicz D, Chrobak A, Gmyrek GB, et al. Diagnostic accuracy of interleukin-6 levels in peritoneal fluid for detection of endometriosis Arch Gynecol Obstet. 2013;288(4):805-814.

10. Birt JA, Nabli H, Stilley JA, Windham EA, Frazier SR, Sharpe-Timms KL. Elevated peritoneal fluid TNF-alpha incites ovarian early growth response factor 1 expression and downstream protease mediators: a correlation with ovulatory dysfunction in endometriosis. Reprod Sci. 2013;20(5):514-523.
11. Kajitani T, Maruyama T, Asada $\mathrm{H}$, et al. Possible involvement of nerve growth factor in dysmenorrhea and dyspareunia associated with endometriosis. Endocr J. 2013;60(10):1155-1164.

12. Vercellini P, Crosignani PG, Abbiati A, Somigliana E, Vigano P, Fedele L. The effect of surgery for symptomatic endometriosis: the other side of the story. Hum Reprod Update. 2009;15(2):177-188.

13. Deguara CS, Pepas L, Davis C. Does minimally invasive surgery for endometriosis improve pelvic symptoms and quality of life? Curr Opin Obstet Gynecol. 2012;24(4):241-244.

14. Lessey BA. Medical management of endometriosis and infertility. Fertil Steril. 2000;73(6):1089-1096.

15. Greene AD, Lang SA, Kendziorski JA, Sroga-Rios JM, Herzog TJ, Burns KA. Endometriosis: where are we and where are we going? Reproduction. 2016;152(3):R63-R78.

16. Hansen RR, Nasser A, Falk S, et al. Chronic administration of the selective $\mathrm{P} 2 \times 3, \mathrm{P} 2 \times 2 / 3$ receptor antagonist, A-317491, transiently attenuates cancer-induced bone pain in mice. Eur J Pharmacol. 2012;688(1-3): $27-34$.

17. Cantin L, Bayrakdarian M, Buon C, et al. Discovery of $\mathrm{P} 2 \times 3$ selective antagonists for the treatment of chronic pain. Bioorg Med Chem Lett. 2012;22(7):2565-2571.

18. Sharp CJ, Reeve AJ, Collins SD, et al. Investigation into the role of $\mathrm{P} 2 \mathrm{X}(3) / \mathrm{P} 2 \mathrm{X}(2 / 3)$ receptors in neuropathic pain following chronic constriction injury in the rat: an electrophysiological study. BrJ Pharmacol. 2006;148(6):845-852.

19. Gever JR, Soto R, Henningsen RA, et al. AF-353, a novel, potent and orally bioavailable $\mathrm{P} 2 \times 3 / \mathrm{P} 2 \times 2 / 3$ receptor antagonist. Br J Pharmacol. 2010;160(6):1387-1398.

20. Hu F, Zhang Y, Du Y, Yuan H. Nimodipine loaded lipid nanospheres prepared by solvent diffusion method in a drug saturated aqueous system. Int J Pharm. 2008;348(1-2):146-152.

21. Beloqui A, Angeles Solinis M, Rodriguez-Gascon A, Almeida AJ, Preat V. Nanostructured lipid carriers: promising drug delivery systems for future clinics. Nanomed Nanotechnol. 2016;12(1):143-161.

22. Ebrahimi HA, Javadzadeh Y, Hamidi M, Jalali MB. Development and characterization of a novel lipohydrogelnanocarrier: repaglinide as a lipophilic model drug. J Pharm Pharmacol. 2016;68(4): 450-458.

23. Zhao M, Sun Y, Fu G, et al. Gene therapy of endometriosis introduced by polymeric micelles with glycolipid-like structure. Biomaterials. 2012; 33(2):634-643.

24. He S, Li Y, Yan J, et al. Ternary nanoparticles composed of cationic solid lipid nanoparticles, protamine, and DNA for gene delivery. Int $J$ Nanomedicine. 2013;8:2859-2869.

25. Chai G, Xu Y, Chen S, et al. Transport mechanisms of solid lipid nanoparticles across Caco-2 cell monolayers and their related cytotoxicology. ACS Appl Mater Interfaces. 2016;8(9):5929-5940.

26. Hu F, Ren G, Yuan H, Du Y, Zeng S. Shell cross-linked stearic acid grafted chitosan oligosaccharide self-aggregated micelles for controlled release of paclitaxel. Colloid Surf B Interfaces. 2006;50(2): 97-103.

27. Meng T, Liu J, Wen L, et al. Multi-cycle chemotherapy with the glycolipid-like polymeric micelles evade cancer stem cell enrichment in breast cancer therapy. Oncotarget. 2016;7(45):72978-72989.

28. Yi H, Wu J, Du Y, et al. Effect of anionic PEGylated polypeptide on gene transfection mediated by glycolipid conjugate micelles. $\mathrm{Mol}$ Pharm. 2015;12(4):1072-1083.

29. Liu J, Liu X, Duan K, Zhang Y, Guo S. The expression and functionality of transient receptor potential vanilloid 1 in ovarian endometriomas. Reprod Sci. 2012;19(10):1110-1124.

30. Hwang T, Aljuffali IA, Hung C, Chen C, Fang J. The impact of cationic solid lipid nanoparticles on human neutrophil activation and formation of neutrophil extracellular traps (NETs). Chem Biol Interact. 2015; 235:106-114.

31. Sun Y, Che X, Zhu L, et al. Pigment epithelium derived factor inhibits the growth of human endometrial implants in nude mice and of ovarian endometriotic stromal cells in vitro. PLoS One. 2012;7: e452239. 
32. Jarvis MF, Burgard EC, McGaraughty S, et al. A-317491, a novel potent and selective nonnucleotide antagonist of $\mathrm{P} 2 \mathrm{X}(3)$ and $\mathrm{P} 2 \mathrm{X}(2 / 3)$ receptors, reduces chronic inflammatory and neuropathic pain in the rat. Proc Natl Acad Sci US A. 2002;99(26):17179-17184.
33. You J, Wang Z, Du Y, et al. Specific tumor delivery of paclitaxel using glycolipid-like polymer micelles containing gold nanospheres. Biomaterials. 2013;34(18):4510-4519. 


\section{Supplementary material}

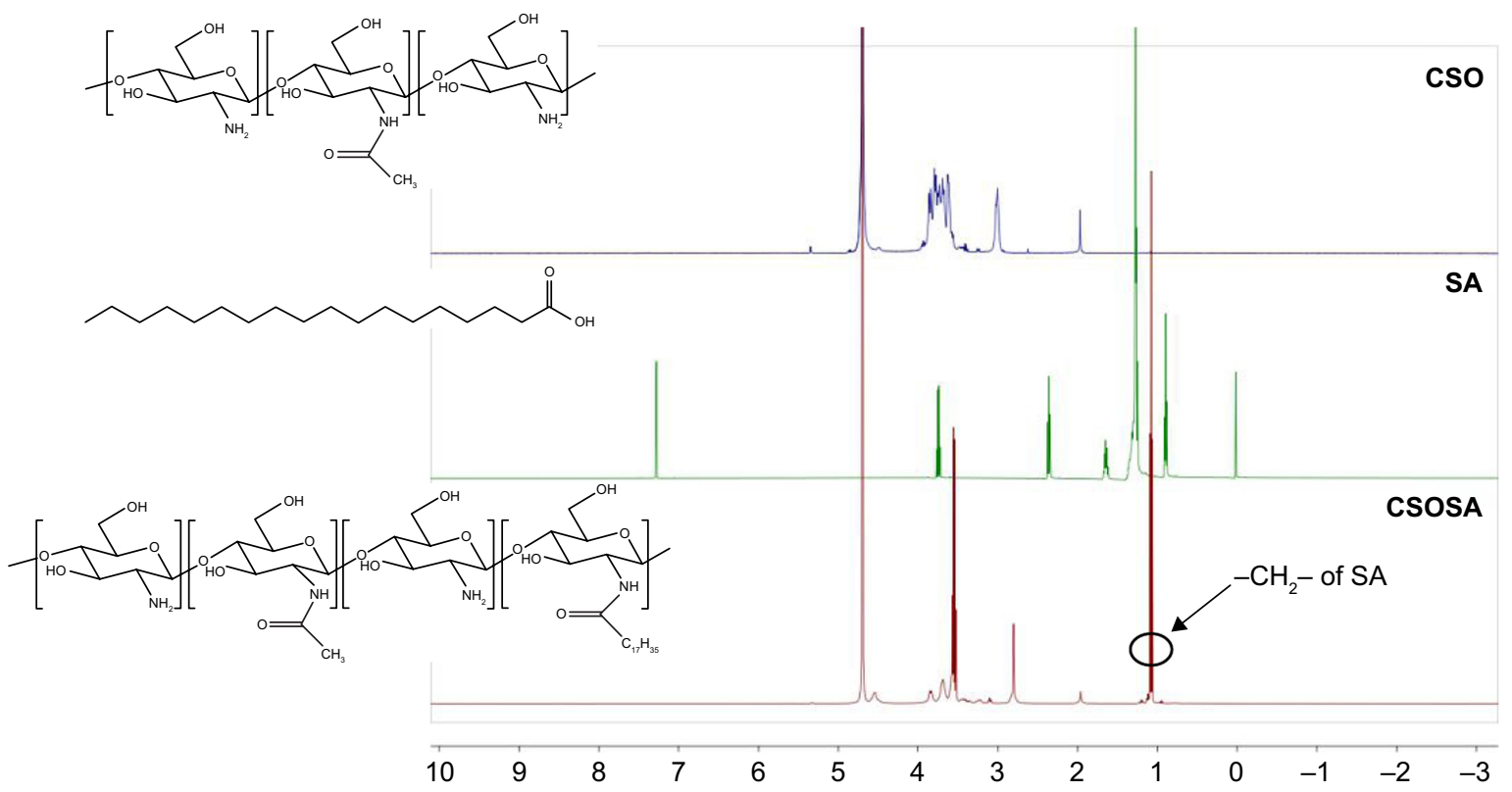

Figure SI Chemical structure verification of CSOSA polymer. 'H NMR spectra of CSO, SA and CSOSA.

Abbreviations: CSOSA, chitosan oligosaccharide-g-stearic acid; 'H NMR, proton nuclear magnetic resonance; CSO, chitosan oligosaccharide; SA, stearic acid.

\section{Publish your work in this journal}

The International Journal of Nanomedicine is an international, peerreviewed journal focusing on the application of nanotechnology in diagnostics, therapeutics, and drug delivery systems throughout the biomedical field. This journal is indexed on PubMed Central, MedLine, CAS, SciSearch $®$, Current Contents $₫ /$ Clinical Medicine,
Journal Citation Reports/Science Edition, EMBase, Scopus and the Elsevier Bibliographic databases. The manuscript management system is completely online and includes a very quick and fair peer-review system, which is all easy to use. Visit http://www.dovepress.com/ testimonials.php to read real quotes from published authors. 